Chirurg 2021 · 92:395-396

https://doi.org/10.1007/s00104-021-01386-w

Angenommen: 22. Februar 2021

Online publiziert: 31. März 2021

(c) Springer Medizin Verlag GmbH, ein Teil von Springer Nature 2021

Redaktion

U. Settmacher, Jena

Utz Settmacher

Klinik für Allgemein-, Viszeral- und Gefäßchirurgie, Universitätsklinikum Jena, Jena, Deutschland

\title{
ERAS - beschleunigte Genesung nach Operationen
}

zum Thema ERAS besprechen und sie so auf den aktuellen Stand der Diskussion bringen und Problemfelder aufzeigen.

Im ersten Beitrag bespricht $A$. Weimann die perioperative Nahrungssupplementation. Der Ernährungszustand des Patienten vor einer Operation hat prognostische Relevanz. Ein Punkt auf den ERAS eingeht und ebenso den frühen Kostaufbau nach der Operation anstrebt. Es wird vorgestellt, wer insbesondere profitiert. Zum Thema „Immunonutrition“ wird ebenfalls Stellung bezogen.

Im zweiten Beitrag analysiert W. Schwenk die aktuelle Studienlage zu ERAS insbesondere in der Viszeral-, Thorax- und Gefäßchirurgie sowie nach orthopädischen Operationen. In allen Patientengruppen verkürzte ERAS die stationäre Verweildauer, ohne dass es zu häufigeren stationären Wiederaufnahmen kam. ERAS senkte unabhängig vom Zugangsweg das Risiko einer postoperativen Komplikation um 30-35\%. Die Rate von Reinterventionen oder Reoperationen wurde durch ERAS nicht beeinflusst.

\section{) Das intraoperative Flüssigkeitsmanagement sollte individuell angepasst sein}

Wichtigste Partner in der Durchsetzung von ERAS sind die Anästhesisten. Im dritten Beitrag beschreiben J. Renner et al., was aus anästhesiologischer Sicht insbesondere hinsichtlich des Flüssigkeitsmanagements $\mathrm{zu}$ beachten ist. Besonders zur Durchsetzung von ERAS sollte das intraoperative Flüssigkeitsmanagement an den individuellen Be- darf des Patienten angepasst sein. Als Grundsatz einer differenzierten Flüssigkeitstherapie wird formuliert, dass eine Volumen- oder Flüssigkeitssubstitution überhaupt nur dann in Betracht gezogen werden sollte, wenn Patienten sowohl verbesserte Perfusionsverhältnisse benötigen als auch positiv auf Flüssigkeitsgabe ansprechen, mit dem Ziel eine Hypovolämie und eine $\mathrm{Hy}$ pervolämie zu vermeiden. Weiter wird auf die Modifikation der Medikation und den Einsatz des Epiduralkatheters eingegangen.

S. Seyfried et al. berichten im nächsten Beitrag über die Implementierung des ERAS $^{\circledR}$-Konzepts in ihrer Klinik. Es wird versucht $\mathrm{zu}$ zeigen, dass die perioperative Behandlungsqualität durch Implementation eines zertifizierten ERAS $^{\circledR}$ Programmes im Setting eines bereits seit 2008 etablierten „Fast-track“-Pfades noch zu steigern ist. Für die Implementierung wurde ein professionelles externes Beratungsteam genutzt. Extrem wichtig ist die Einbeziehung aller Berufsgruppen des Krankenhauses. Dies wird von den Autoren als essenziell angesehen, um langfristig Erfolg zu haben und einen Denkprozess im gesamten Krankenhaus anzustoßen.

Der letzte Beitrag von S. Schüle et al. widmet sich den Perspektiven von ERAS und dessen Weiterführung in einen teilambulanten Bereich unter Nutzung moderner Konzepte der Digitalisierung. Die Integration von ERAS in ein Smart Hospital mit Anschlussversorgung in Smart Homes „auf Zeit“ wird vorgestellt. Hierzu wird unter Nutzung einer individuell konfigurierten Onlinewissensplattform ein Großteil der Informa- 
tionsübermittlung vom ambulanten in den präambulanten Bereich verschoben, wodurch Patienten bereits beim Erstkontakt mit der Klinik besser vorbereitet sind. Nach kurzem stationärem Aufenthalt erfolgt die Entlassung in die Serviced Appartments des smarten Quartiers, wo eine stressfreie Erholung in wohnlicher Umgebung möglich ist. Die weitere Rehabilitation dort erfolgt unter virtueller Begleitung nach individuellem Zeitplan „on demand“.

Ich wünsche Ihnen Freude beim Lesen<smiles>CCCCCCCCCCCCCCCC</smiles>

Prof. Dr. Utz Settmacher

\section{Korrespondenzadresse}

\section{Prof. Dr. Utz Settmacher}

Klinik für Allgemein-, Viszeral- und

Gefäßchirurgie, Universitätsklinikum Jena Erlanger Allee 101, 07740 Jena, Deutschland

Utz.Settmacher@med.uni-jena.de

Interessenkonflikt. U. Settmacher gibt an, dass kein Interessenkonflikt besteht.

\section{Neuerscheinungen im April/Mai 2021}

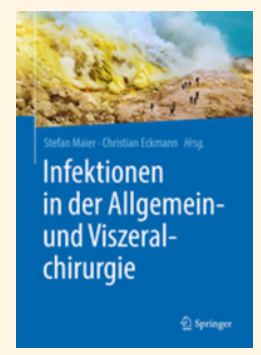

Infektionen in der Allgemein- und Viszeralchirurgie

S. Maier, C. Eckmann (Hrsg.) eBook 42,99 EUR, Softcover 54.99 EUR, ISBN 978-3-662-62507-1

Entstehung, Diagnostik und Behandlung des breiten Spektrums allgemein- und viszeralchirurgischer Infektionen sind detailliert und praxisorientiert von anerkannten Experten beschrieben; damit erhält der Leser eine fundierte Grundlage, sich als primär behandelnder Arzt kompetent in der meist interdisziplinären Therapiediskussion zu positionieren. Die Herausgeber und viele der Autoren gehören der Arbeitsgruppe Allgemein- und Viszeralchirurgische Infektionen der DGAV an.

https://www.springer.com/de/book/9783662625071

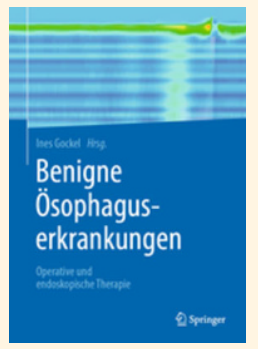

\section{Benigne Ösophaguserkrankungen.}

Operative und endoskopische Therapie

I. Gockel (Hrsg.) eBook 66,99 EUR, Hardcover 84,99 EUR, ISBN 978-3-66260454-0

Experten für die jeweilige Thematik stellen systematisch den Wissensstand zur Pathogenese der verschiedenen benignen Erkrankungen der Speiseröhre, das empfohlene diagnostische Vorgehen und die Therapiemöglichkeiten dar. Detailliert beschreiben sie Auswahl, Durchführung und Ergebnisse der einzelnen endoskopischen und operativen Verfahren, zahlreiche Abbildungen veranschaulichen das Vorgehen. https://www.springer.com/de/book/9783662604540

\section{Evidenzbasiertes perioperatives Management in der Viszeral-} chirurgie.

Leitlinien, Empfehlungen und Studienlage

R. Grundmann, E.S. Debus (Hrsg.) Softcover ca. 54,99 EUR, ISBN 978-3-66262847-8

Evidenzbasiertes

perioperatives

Management in

der Viszeralchirurgie

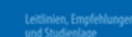

פspinger
Das Buch bildet die Basis für eine evidenzbasierte perioperative Behandlung bei allen häufigen viszeralchirurgischen Operationen. Die einzelnen Maßnahmen (wie Ernährung, Analgesie, perioperative Antibiotikaprophylaxe und Hämotherapie) werden auf der Grundlage der weltweit publizierten Empfehlungen bewertet. Berücksichtigt sind die nationalen und internationalen Leitlinien, die Ergebnisse von Metaanalysen, Cochrane-Reviews und anderen randomisierten Studien sowie weitere verfügbare Informationen, die nicht in den Leitlinien stehen.

Ganz aktuell: ein Kapitel zum perioperativen Management bei COVID19-Patienten!

https://www.springer.com/de/book/9783662628478 